\title{
Treatment of ocular myasthenia with corticotrophin
}

\author{
F. B. GIBBERD, F. NAVAB, AND C. L. SMITH \\ From the Neurological Clinic, Westminster Hospital, London
}

SUMMARY Nine patients with severe myasthenia gravis, including ocular symptoms, who did not respond to anticholinesterase therapy were given prolonged courses of corticotrophin. In all patients except one there was an initial deterioration, sometimes necessitating assisted respiration, followed by a marked improvement which persisted for many weeks and occasionally months. It is concluded that corticotrophin should be continued for at least two weeks as the onset of the remission might be delayed. Repeated courses or small maintenance doses prolonged the remission. Fluid retention and hypokalaemia were common unwanted effects but necessitated stopping the treatment in only one patient.

Autoimmune phenomena have a role in myasthenia gravis (Simpson, 1960; White and Marshall, 1962; van der Geld, Feltkamp, and Oosterhuis, 1964) and there is an association between myasthenia gravis and diseases with autoimmune features (Piemme, 1964). Corticotrophin therapy for myasthenia gravis was first used by Torda and Wolff (1949 and 1951) and Millikan and Eaton (1951). In generalized myasthenia courses of corticotrophin for five to 10 days have been noted to produce a deterioration followed by improvement which may persist for several months (Freydberg, 1960; Grob and Namba, 1966; Osserman and Genkins, 1966; Reis, Liljestrand, and Matell, 1966). Recently, Cape and Utterback (1969) have suggested that maintenance injections weekly or twice monthly may prolong the remission. In the only report on the treatment of ocular myasthenia by Mount (1964a and b), no significant improvement was detected after an eight day course of corticotrophin. The present study was performed to see whether an extended course of corticotrophin could produce an improvement in myasthenia in patients with ocular symptoms.

\section{METHODS}

All the patients with myasthenia attending a general neurological clinic were reviewed. Those with ocular myasthenia who were not obtaining full recovery with standard anticholinesterase treatment and in whom there was no contraindication to corticotrophin were admitted to the trial. None had previously received corticosteroids while suffering from myasthenia. During the initial few days of their hospital admission their treatment was kept constant and they were assessed clinically. Edrophonium was used to confirm the diagnosis of myasthenia but produced little improvement when on full anticholinesterase treatment. In all patients, except case 8 , the clinical state had remained unchanged for over three months. During the time they were given corticotrophin by intramuscular injection they were assessed clinically and biochemically. As the patients' previous conditions were stable the assessment could be made by comparison with their pretreatment state.

\section{RESULTS}

The important clinical findings are given in Table 1 and the results of treatment in Table 2.

\section{DISCUSSION}

Corticotrophin in this series of patients with myasthenia produced well recognized initial deterioration followed by improvement. The one patient in whom there was no deterioration improved only slightly. The initial deterioration can be very sudden and may be severe, and four patients required assisted respiration.

Myasthenic symptoms were not affected appreciably by the unwanted effects of hypokalaemia and fluid retention, which were related to corticotrophin therapy.

Similar results were obtained with soluble corticotrophin, corticotrophin gel or carboxymethylcellulose, and tetracosactrin zinc phosphate. Previous 
TABLE 1

CLINICAL STATE

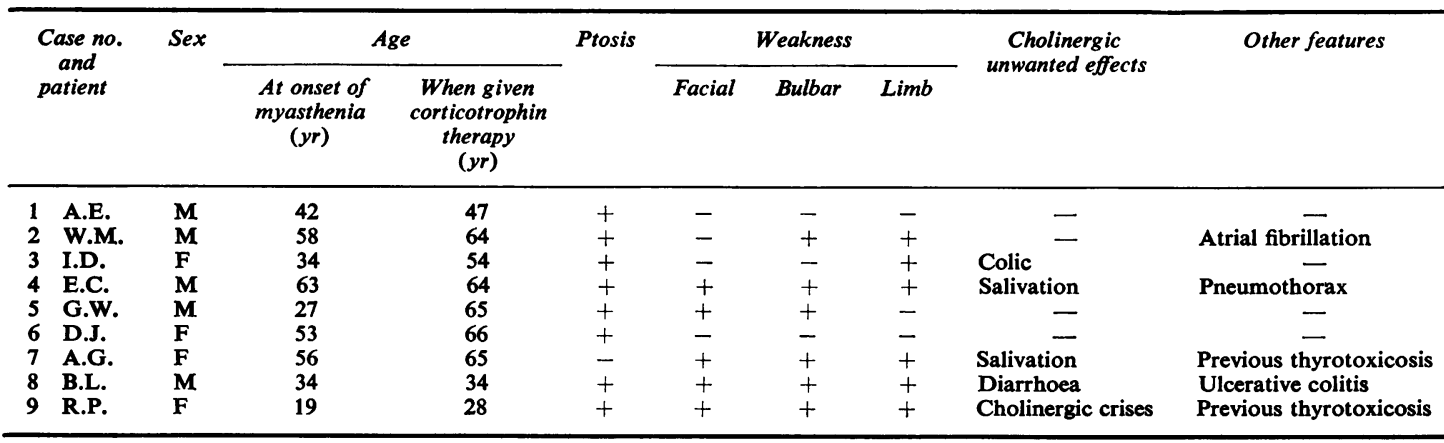

All patients had diplopia.

reports (Freydberg, 1960; Grob and Namba, 1966; Osserman and Genkins, 1966; Reis et al., 1966) of short courses of corticotrophin therapy suggested that the initial deterioration occurred during treatment and the symptoms improved after injections were stopped. This cannot be correct as, in the present trial, treatment was continued throughout the period of improvement.

Corticotrophin undoubtedly improved the ocular myasthenia in the same way as the rest of the musculature in all the patients except one, as would have been expected from the previous reports on corticotrophin in generalized myasthenia (Freydberg, 1960; Grob and Namba, 1966; Osserman and Genkins, 1966; Reis et al., 1966). Mount (1964a and b) reported a multicentred controlled trial of corticotrophin in 43 patients with ocular myasthenia, 2 21 of whom received $40 \mathrm{u}$. corticotrophin gel for six days, then $30 \mathrm{u}$. for one day, and finished on the?

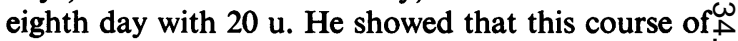
corticotrophin produced no demonstrable improve-ment or deterioration in the signs and symptoms of $-\overrightarrow{-}$ patients with ocular myasthenia two days and ofe을 and three months after treatment. In view of the many reports of an initial worsening before improve-T ment, the assessments were not made at the optimalo time and an eight day course would appear to be too short.

In the present study, the improvement starte $\overrightarrow{0}$ about one week after treatment had been instituted 2 and then continued, becoming maximal at about the 14th day. In some the improvement continued for

TA BLE 2

CORTICOTROPHIN TREATMENT

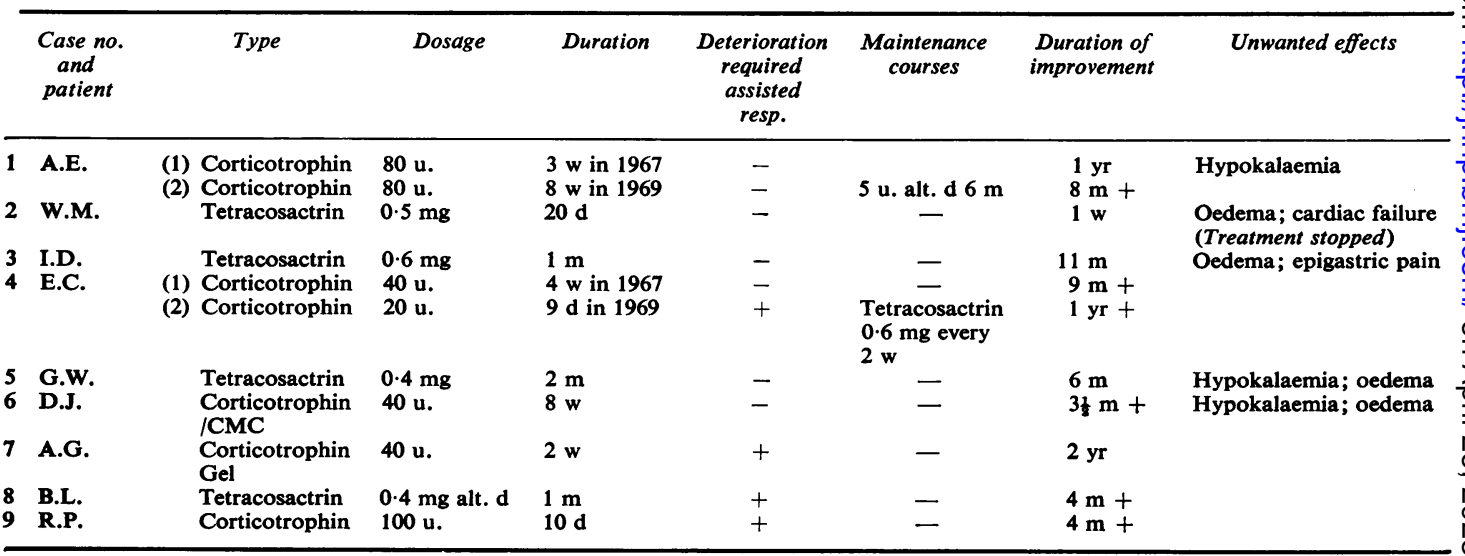


a further week. The clinical condition then usually remained static for several weeks and after a variable period of weeks or months gradually reverted to its previous level.

Cape and Utterback (1969) treated five patients with about $1,000 \mathrm{u}$. corticotrophin over 10 days. In three patients there was deterioration within the first week. Improvement followed in all patients within two weeks and was maintained for at least two months. Within six months they had all relapsed and three were given repeated courses with a similar incidence of initial deterioration. Maintenance therapy of $100 \mathrm{u}$. weekly or fortnightly was given to two patients after repeated intensive courses of corticotrophin and to one patient after a single intensive course. All three maintained their improvement during the period of 16 to 23 months during which they were followed. The dosage was not varied to see if smaller doses or wider intervals would have had a similar effect. Our patients were given corticotrophin for much longer than in most of the published reports and this probably explains why their improvement was maintained for such long periods. We would support the suggestion of Cape and Utterback (1969) that maintenance therapy should be tried for those patients who improve on corticotrophin but relapse when it is stopped.

Complications resulting from corticotrophin therapy occurred in some patients. Of the four patients who had ankle oedema (cases $2,3,5,6$ ) one developed congestive cardiac failure. All these cases responded to diuretics during the corticotrophin therapy, except that in the patient with congestive cardiac failure corticotrophin had to be stopped. Three patients developed marked lassitude and mild generalized non-myasthenic weakness associated with hypokalaemia. Potassium supplements were often insufficient to restore the serum level. Low potassium levels did not interfere with the patient's improvement in myasthenic symptoms, as serum levels could continue to fall while myasthenic features regressed.

Tetracosactrin zinc sulphate seemed to result in a more severe degree of hypokalaemia and fluid retention than previous preparations. Injections of tetracosactrin depot should probably be limited to alternate days to avoid accumulation and in three cases where this was done the unwanted effects were reduced. Prophylactic diuretics should not be given because this would exacerbate the hypokalaemia but potassium supplements should be given routinely. Repeated courses and maintenance can be given.

In almost all patients corticotrophin therapy was beneficial and is recommended in ocular myasthenia or generalized myasthenia not responding adequately to conventional treatment.
CONCLUSIONS

Corticotrophin compounds may be used to treat severe myasthenia which is insufficiently controlled by anticholinesterases. A deterioration should be anticipated during the first 10 days which may necessitate assisted respiration. Massive dosage is unnecessary. Treatment should be continued for at least two weeks as the onset of the remission may be delayed for this length of time. Maintenance therapy may prolong remissions.

Side-effects such as hypokalaemia and fluid retention occur and the long acting preparations may be given on alternate days.

We would like to thank Dr. R. I. S. Bayliss for permission to report case 9 .

\section{REFERENCES}

Cape, C. A., and Utterback, R. A. (1969). Treatment of myasthenia gravis with adrenocorticotropic hormone (ACTH): massive short-term and maintenance treatment. J. Neurol. Neurosrug. Psychiat., 32, 290-296.

Freydberg, L. D. (1960). The place of corticotropin in the treatment of myasthenia gravis. Ann. intern. Med., 52, 806-818.

Grob, D., and Namba, T. (1966). Corticotrophin in generalized myasthenia gravis. Effect of short, intensive courses. J. Amer. med. Ass., 198, 703-707.

Millikan, C. H., and Eaton, L. M. (1951). Clinical evaluation of ACTH and cortisone in myasthenia gravis. Neurology (Minnean.), 1, 145-152.

Mount, F. W. (1964a). ACTH for ocular myasthenia. $J$. Amer. med. Ass., 189, 55.

Mount, F. W. (1964b). Corticotropin in treatment of ocular myasthenia; a controlled clinical trial. Arch. Neurol. (Chic.), 11, 114-124.

Osserman, K. E., and Genkins, G. (1966). Studies in myasthenia gravis. Short-term massive corticotrophin therapy. J. Amer. med. Ass., 198, 699-702.

Piemme, T. E. (1964). Myasthenia gravis and auto-immune disease. Review of the literature including a case report of the coexistence of myasthenia and systemic lupus erythematosus. Ann. intern. Med., 60, 130-135.

von Reis, G., Liljestrand, §., and Matell, G. (1966). Treatment of severe myasthenia gravis with large doses of ACTH. Ann. N.Y. Acad. Sci., 135, 409-416.

Simpson, J. A. (1960). Myasthenia gravis: a new hypothesis. Scot. med.J., 5, 419-436.

Torda, C., and Wolff, H. G. (1949). Effect of adrenocorticotrophic hormone on neuromuscular function in patients with myasthenia gravis. J. clin. Invest., 28, 12281235.

Torda, C., and Wolff, H. G. (1951). Effects of administration of adreno-corticotropic hormone (ACTH) in patients with myasthenia gravis. Arch. Neurol. Psychiat. (Chicago), 66, 163-170.

van der Geld, H., Feltkamp, T. E. W., and Oosterhuis, H. J. G. H. (1964). Reactivity of myasthenia gravis serum $\gamma$-globulin with skeletal muscle and thymus demonstrated by immunofluorescence. Proc. Soc. exp. Biol. (N.Y.), 115, 782-785.

White, R. G., and Marshall, A. H. E. (1962). The autoimmune response in myasthenia gravis. Lancet, 2, 120123. 